07,10

\title{
Фактор предпочтения базисной краевой дислокационной петли в цирконии. Численный анализ
}

\author{
(ㄱ А.В. Бабич, В.Ф. Клепиков, П.Н. Остапчук
}

Институт электрофизики и радиационных технологий НАН Украины, Харьков, Украина

E-mail: ostapchuk@kipt.kharkov.ua

Поступила в Редакцию 8 августа 2020 г.

В окончательной редакции 8 августа 2020 г.

Принята к печати 18 августа 2020 г.

Недавние численные расчеты коэффициентов диффузии радиационных точечных дефектов в гексагональных кристаллах показали, что основное предположение теории радиационного роста циркония (DAD diffusional anisotropy difference) не выполняется. Таким образом, упругая идеология (EID - elastic interaction difference), основанная на концепции фактора предпочтения стока остается актуальной. В этой связи численно (методом конечных разностей) посчитан фактор предпочтения базисной краевой петли циркония в тороидальном резервуаре с учетом упругой анизотропии гексагонального кристалла. Тороидальная геометрия резервуара позволяет провести расчеты для петли любого размера и без какой-либо коррекции упругого поля в ее области влияния. Получены зависимости фактора предпочтения петли от ее радиуса и природы при различных плотностях стоков. Показана существенная роль формы граничного условия на внешней поверхности резервуара. Обозначены перспективы дальнейших исследований в построении теории радиационного роста циркония на основе упругой идеологии.

Ключевые слова: точечный дефект, базисная дислокационная петля, фактор предпочтения, цирконий, радиационный рост.

DOI: 10.21883/FTT.2020.12.50212.165

\section{1. Введение}

Как известно, концепция предпочтительного поглощения внутренними протяженными дефектами кристалла (стоками) радиационных точечных дефектов (ТД) определенного сорта (вакансий или собственных междоузельных атомов - CMA) считается центральным элементом теории радиационно-индуцированной деформации. Такая особенность стоков (пор, дислокационных петель, сетки дислокаций) обуславливает некоторую ассиметрию диффузионных потоков ТД на них и ведет к эволюции микроструктуры материала и его макроскопической деформации (например, радиационному распуханию и росту). Исторически, теория радиационной деформации строилась главным образом для объяснения явления вакансионного распухания кубических металлов и сплавов [1-3]. Они, как правило, моделируются упруго- и диффузионно-изотропной средой, а причиной разделения потоков ТД считается более сильное упругое взаимодействие междоузлий с краевыми компонентами дислокаций по сравнению с вакансиями. Говорят, что дислокации эффективнее поглощают СМА (имеют к ним предпочтение - „bias“, „преференс“"), а остающиеся в избытке вакансии поглощаются порами, обуславливая их рост, что в конечном итоге приводит к увеличению объема облучаемого материала. Однако попытки применить упругую идеологию к ГПУ-металлам, в частности для объяснения радиационного роста (РP) циркония, успеха пока не имели. Явление РР сопровождается изменением формы материала без приложения внешней нагрузки и без заметного изменения объема. Цирконий в процессе роста расширяется в $\langle a\rangle$-направлении и сужается вдоль $\langle c\rangle$-оси [4,5]. Такое возможно, если, например, на базисных плоскостях зарождаются и растут вакансионные петли, „съедающие“ кристалл вдоль $\langle c\rangle$-оси, а на призматических - междоузельные, образуя дополнительные экстраплоскости в $\langle a\rangle$-направлении [6]. Однако механизм роста вакансионных петель пока не ясен. Согласно классической упругой идеологии [7] „bias“-фактор петли не зависит от ее природы. В таком случае зарождение, а тем более рост вакансионных петель труднообъясним. В литературе имеется альтернативный вариант упругой идеологии (EID elastic interaction difference). Это идеология анизотропной диффузии ТД, характерная для ГПУ-кристаллов (DAD - diffusional anisotropy difference) [8,9]. Основное предположение соответствующей теории следующее $D_{i}^{a} / D_{i}^{c}>D_{v}^{a} / D_{v}^{c}$, где $D_{m \text {, }}^{a}-$ коэффициент диффузии ТД $m$-го сорта в базисной плоскости циркония, $D_{m}^{c}-$ аналогичная величина в $\langle c\rangle$-направлении индексы $v, i$ относятся к вакансиям и СМА соответственно). Тогда оказывается, что прямолинейные дислокации в базисной и призматической плоскостях переползают в противоположных направлениях, т.е. имеет место разделение диффузионных потоков ТД между этими плоскостями: вакансии преимущественно идут в базисную плоскость циркония, а междоузлия в призматическую. Тем самым возникает принципиальная возможность роста базисных 
вакансионных петель. Однако экспериментальных подтверждений этого неравенства на сегодняшний день нет. Более того, недавние численные расчеты [10] показали следующее. Вакансии и СМА действительно показывают анизотропную диффузию преимущественно параллельно базисной плоскости $\left(D_{i, v}^{a} / D_{i, v}^{c}>1\right)$, однако неравенство в области реакторных температур $T<800 \mathrm{~K}$ как раз обратное $D_{i}^{a} / D_{i}^{c}<D_{v}^{a} / D_{v}^{c}$, и авторы [10] сомневаются в возможности объяснения наблюдаемого роста циркония теорией анизотропной диффузии DAD. Поэтому остается только концепция упругого „bias“-фактора. В этой связи в работе численно рассчитывается фактор предпочтения базисной краевой петли разной природы и обсуждается возможность дальнейшего применения упругой идеологии для объяснения РР циркония.

Если известно фиктивное распределение объемных сил $f_{i}^{S}$, создающих в упругой среде такие же напряжения, как и реальный источник $S$, то энергию взаимодействия между двумя системами внутренних напряжений $S\left(\mathbf{u}^{S}, u_{i j}^{S}, \sigma_{i j}^{S}\right)$ и $T\left(\mathbf{u}^{T}, u_{i j}^{T}, \sigma_{i j}^{T}\right)$, согласно Эшелби [11], можно представить интегралом вида

$$
E_{\mathrm{int}}(S, T)=-\int f_{i}^{S} u_{i}^{T} d V
$$

который берется по области, содержащей только источник системы $S$. Пусть это точечный дефект (ТД) дипольного типа, описываемый в теории упругости объемным распределением дипольных сил без момента

$$
f_{i}^{S}(\mathbf{r})=-P_{i j} \nabla_{j} \delta(\mathbf{r}-\mathbf{a}), \quad P_{i j}=P_{j i} .
$$

Тогда для энергии упругого взаимодействия между ТД и системой напряжений $T$ имеем

$$
E_{\mathrm{int}}(\mathbf{r})=-\int f_{i}\left(\mathbf{r}^{\prime}\right) u_{i}^{T}\left(\mathbf{r}^{\prime}\right) d \mathbf{r}^{\prime}=-P_{i j} u_{i j}^{T}(\mathbf{r}),
$$

где $u_{i j}^{T}(\mathbf{r})$ - поле деформаций, вызванное системой $T$ в точке $\mathbf{r}=\mathbf{a}$ нахождения ТД. Система координат при этом связана с источником внутренних напряжений $T$. Обратим внимание, что он никак не конкретизирован. Это может быть другой ТД либо дислокационная петля. Но в любом случае надо уметь вычислять поле деформаций, создаваемое этим источником в среде, моделирующей кристалл конкретной сингонии. Если упругий диполь обладает осевой симметрией, то он характеризуется тензором вида

$$
P_{i j}=P_{0} \delta_{i j}+P_{1}\left(l_{i} l_{j}-1 / 3 \delta_{i j}\right),
$$

где $\mathbf{l}$ - единичный вектор оси диполя [12]. Будем считать, что для ГПУ-сингонии она совпадает с $\langle c\rangle$-осью кристалла. Тогда в декартовой систе координат с осью $z$ вдоль $\langle c\rangle$-оси ГПУ-кристалла тензор $P_{i j}$ имеет только диагональные компоненты, которые в сокращенном описании можно представить в виде $P_{i}=P(1,1, \varepsilon), P=P_{a}$, $\varepsilon=P_{c} / P_{a}$, где $P_{a}$ и $P_{c}-$ мощности силовых диполей в $\langle a\rangle$ - и $\langle c\rangle$-направлениях соответственно. Значение параметра $\varepsilon=1$ соответствует центру дилатации $\left(P_{1}=0\right)$. Отметим, что для дефекта, описываемого плотностью сил (2), в кристалле с не кубической симметрией величины $P_{0}$ и $P_{1}$ простого физического смысла не имеют. Поэтому в работах $[13,14]$ по аналогии с $P_{i}=P(1,1, \varepsilon)$ были введены диполи смещений $Q_{i}=Q(1,1, \delta), Q=Q_{a}$, $\delta=Q_{c} / Q_{a}$, связанные с силовыми диполями соотношением $P_{i}=C_{i j} Q_{j}$. Здесь $C_{i j k}-$ упругие модули кристалла. При этом считалось, что изменение объема конечного кристалла $\Delta V$, обусловленное точечным дефектом, связано с диполями смещений соотношением $\Delta V=Q(2+\delta)$. Тогда для ГПУ-кристалла имеем

$$
\begin{aligned}
E_{\text {int }}(\mathbf{r})= & -\Delta V \frac{C_{11}+C_{12}+\delta(\varepsilon) C_{13}}{2+\delta(\varepsilon)} \\
& \times\left[S p u_{i j}^{T}(\mathbf{r})-(1-\varepsilon) u_{33}^{T}(\mathbf{r})\right] \\
\delta(\varepsilon)= & \frac{\varepsilon\left(C_{112}+C_{12}\right)-2 C_{13}}{C_{33}-\varepsilon C_{13}} .
\end{aligned}
$$

В случае центра дилатации $(\varepsilon=1)$ и упруго изотропной среды $\left(C_{11}=C_{22}=C_{33}=\lambda+2 \mu ; C_{12}=C_{13}=\lambda\right)$ получаем: $\delta=1$;

$$
E_{\text {int }}(\mathbf{r})=-\Delta V \frac{3 \lambda+2 \mu}{3} S p u_{i j}^{T}(\mathbf{r})=-\Delta V K S p u_{i j}^{T}(\mathbf{r}),
$$

где $K$ - модуль всестороннего сжатия. Таким образом, остается конкретизировать источник внутренних напряжений $T$ и рассчитать поле деформаций, создаваемое этим источником в данной упругой среде.

\section{2. Упругое поле деформаций базисной дислокационной петли гексагонального кристалла}

В качестве источника напряжений $T$ рассмотрим краевую вакансионную петлю радиуса $R$, лежащую в плоскости $z=0$ (базисная плоскость) цилиндрической системы координатах $(r, \varphi, z)$, вектор Бюргерса которой перпендикулярен плоскости петли и имеет только $z$-компоненту $(0,0, b)$ (рис. 1). Вектор нормали к плоскости петли совпадает с положительным направлением оси $z$, являющейся также осью симметрии кристалла. В работах $[15,16]$ методом функций Грина в подходе И.М. Лифшица, Л.Н. Розенцвейга для искомых выше величин были получены следующие аналитические выражения

$$
\begin{aligned}
\operatorname{Spu}_{i j}^{D}(\mathbf{r})= & -\frac{b}{4 \pi} \int_{S_{D}} \frac{d^{2} r^{\prime}}{\left|\mathbf{r}-\mathbf{r}^{\prime}\right|^{3}} \\
& \times\left[\left(1-3 \tau_{3}^{2}\right) Q\left(\tau_{3}^{2}\right)+2 \tau_{3}^{2}\left(1-\tau_{3}^{2}\right) \frac{d Q}{d \tau_{3}^{2}}\right],
\end{aligned}
$$




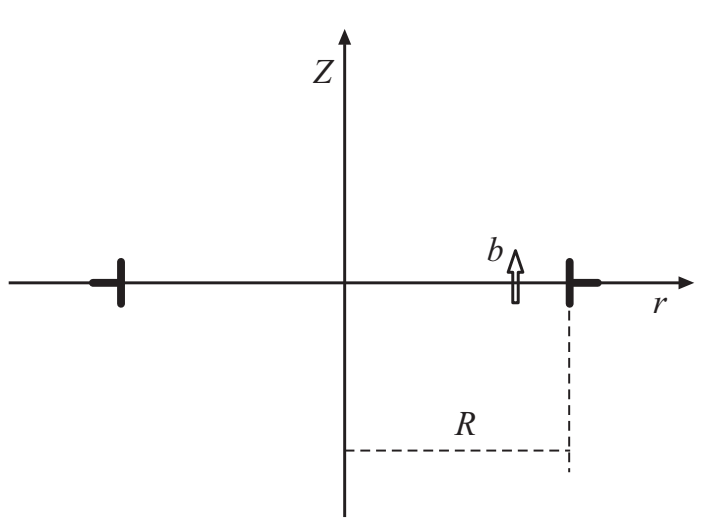

Рис. 1. Вакансионная петля радиуса $R$, лежащая в плоскости $x=0$ цилиндрической системы координатах $(r, \varphi, z)$ с вектором Бюргерса $b$ вдоль оси $z$.

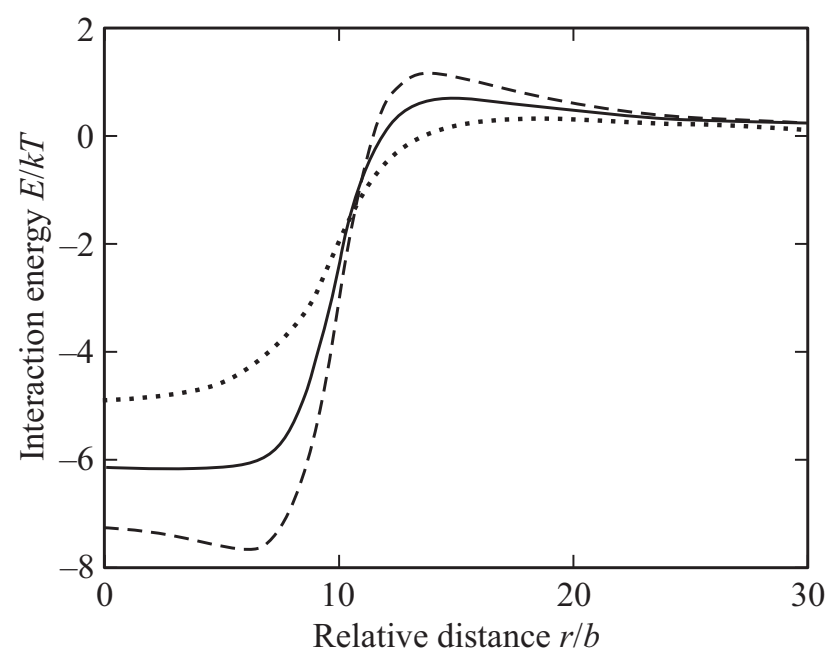

Рис. 2. Зависимость энергии упругого взаимодействия (5) СМА с вакансионной петлей радиуса $R=10 b$ в цирконии от расстояния $\rho=r / b$ в плоскости $z=5 b$ и разных значений параметра $\varepsilon: \varepsilon=0.8$ (точки), $\varepsilon=1$ (сплошная линия), $\varepsilon=1.2$ (пунктир).

$$
\begin{aligned}
& u_{33}^{D}(\mathbf{r})=-\frac{b}{4 \pi} \int_{S_{D}} \frac{d^{2} r^{\prime}}{\left|\mathbf{r}-\mathbf{r}^{\prime}\right|^{3}} \\
& \times\left[\left(1-3 \tau_{3}^{2}\right) \Psi\left(\tau_{3}^{2}\right)+2 \tau_{3}^{2}\left(1-\tau_{3}^{2}\right) \frac{d \Psi}{d \tau_{3}^{2}}\right], \\
& \Psi\left(\tau_{3}^{2}\right)=C_{13} V\left(\tau_{3}^{2}\right)+C_{33} W\left(\tau_{3}^{2}\right), \\
& Q\left(\tau_{3}^{2}\right)= C_{13} K\left(\tau_{3}^{2}\right)+C_{33} W\left(\tau_{3}^{2}\right)+\left(C_{13}+C_{33}\right) V\left(\tau_{3}^{2}\right), \\
& \tau_{3}^{2}=z^{2} /\left|\mathbf{r}-\mathbf{r}^{\prime}\right|^{2} .
\end{aligned}
$$

Функции $K\left(\tau_{3}^{2}\right), \quad W\left(\tau_{3}^{2}\right), \quad V\left(\tau_{3}^{2}\right)$ достаточно громоздкие, поэтому вынесены в Приложение. Интеграл берется по произвольной поверхности $S_{D}$, опирающейся на дислокационную линию (площадь петли). Bсе дальнейшие расчеты будем проводить в безразмерных координатах $r \rightarrow r / b, z \rightarrow z / d,\left|\mathbf{r}-\mathbf{r}^{\prime}\right|^{2}=$ $=r^{2}+z^{2}-2 r r^{\prime} \cos \left(\varphi-\varphi^{\prime}\right)+r^{\prime 2}$. В силу изотропии в базисной плоскости кристалла зависимость от азимутального угла $\varphi$ в (6) отсутствует, поэтому для определенности положим его равным нулю. Характер зависимости безразмерной $\left(E_{\mathrm{int}} / k T\right)$ энергии взаимодействия CMA с вакансионной петлей радиуса $R=10 b$ в цирконии от расстояния $r$ показан на рис. 2 для плоскости $z=5 b$ и разных значений параметра $\varepsilon$. Экспериментальные значения упругих модулей циркония согласно [17] следующие (Mbar): $C_{11}=1.554 ; C_{12}=0.672$; $C_{13}=0.646 ; C_{33}=1.725 ; C_{55}=C_{44}=0.363$. Остальные величины: $T=573 \mathrm{~K}, \Delta V=1.2 \omega, \omega=2.36 \cdot 10^{-29} \mathrm{~m}^{3}$. Видно, что междоузельный атом в виде вытянутого (вдоль $\langle c\rangle$-оси) эллипсоида вращения $(\varepsilon=1.2)$ притягивается (внутренняя область петли) и отталкивается (внешняя) сильнее, чем центр дилатации $(\varepsilon=1)$ и тем более чем сплюснутый сфероид $(\varepsilon=0.8)$. Аналогичным образом взаимодействует с петлей и вакансия, но с поправкой на знак $(\Delta V=-0.6 \omega)$.

\section{3. Фактор предпочтения петли. Постановка задачи}

В случае диффузионной изотропии среды $D_{i k}=D \delta_{i k}$ поток точечных дефектов на дислокационную петлю $J$ находится, путем решения в ее области влияния следующей диффузионной задачи в квазистационарном приближении:

$$
\begin{aligned}
& \omega \operatorname{div} \mathbf{j}(\mathbf{r})=0, \omega \mathbf{j}(\mathbf{r})=-D C(\mathbf{r}) \beta \nabla \mu(\mathbf{r}), \beta \equiv 1 / k_{B} T, \\
& \beta \mu(\mathbf{r})=\ln \left(\frac{C(\mathbf{r})}{C^{e}} \exp \left(\beta E_{\text {int }}(\mathbf{r})\right)\right), J=\iint_{S}[\mathbf{n j}(\mathbf{r})] d \sigma .
\end{aligned}
$$

Здесь $C(\mathbf{r})$ - концентрация мигрирующих ТД; $\mathbf{j}(\mathbf{r})$, $\mu(\mathbf{r})$ - их плотность потока и химпотенциал соответственно; $E_{\text {int }}(\mathbf{r})$ - полученная выше (5), (6) их энергия взаимодействия с петлей; $C^{e}-$ равновесная тепловая концентрация ТД в кристалле в отсутствие поля напряжений $E_{\text {int }}$; интеграл берется по произвольной поверхности, содержащей петлю, $\mathbf{n}-$ внешняя нормаль к ней. Уравнение (7) следует дополнить граничными условиями. Внутренняя поверхность $S_{c}$, как правило, выбирается в виде тороида, содержащего линию дислокации, радиус образующей окружности которого $r_{c}$ имеет смысл радиуса ядра дислокации. Граничное условие на ней имеет вид

$$
\left.C(\mathbf{r}) \exp \left(E_{\text {int }}(\mathbf{r})\right)\right|_{S_{c}}=C_{R}
$$

Оно стандартное и соответствует значению химпотенциала ТД на ядре дислокации $\left.\mu\right|_{S_{c}}=\frac{\omega}{b} P\left(r_{c}, R\right)$, где $P-$ сила, приложенная к единице длины петли и действующая нормально к линии дислокации в ее плоскости. Она определяется линейным натяжением дислокационной линии и обуславливает эффект коалесценции петель одной природы при отжиге [18]. Внешняя поверхность 

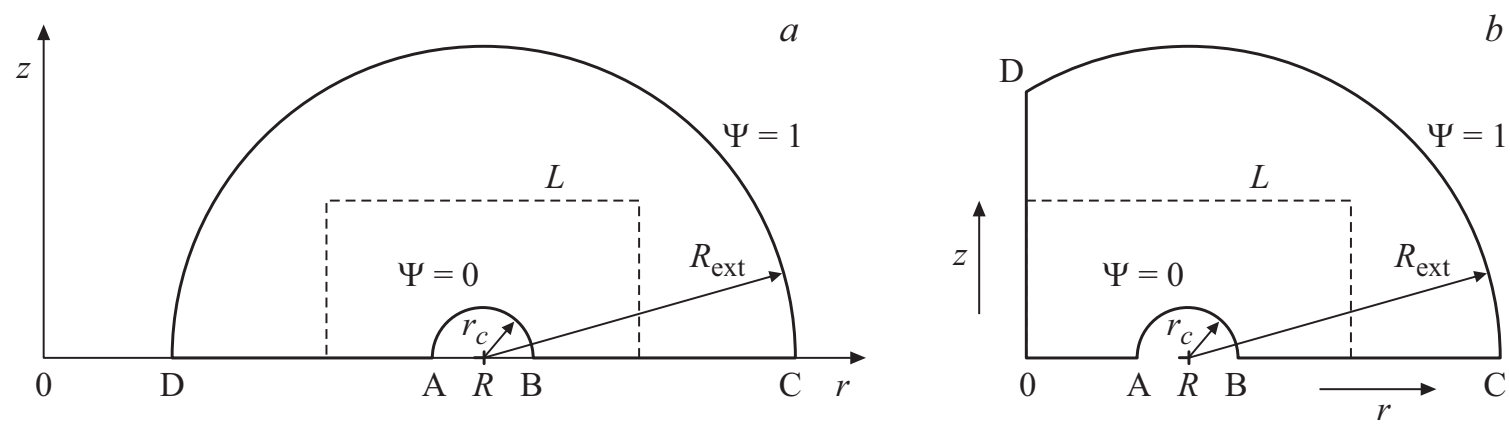

Рис. 3. Система координат для тороидального резервуара: $R>R_{e x t}(a), R<R_{e x t}(b)$.

$S_{\text {ext }}$, выбирается по-разному. Это либо окружность, либо цилиндр, либо соосный с $S_{c}$ тороид $S_{\text {ext }}$, радиус образующей окружности которого $R_{\text {ext }}$ имеет смысл радиуса области влияния петли. Граничное условие на ней обычно [7] формулируется в виде $\left.C(\mathbf{r})\right|_{S_{e x t}}=\bar{C}$, где $\bar{C}$ - средняя концентрация ТД в эффективной среде, моделирующей влияние всего ансамбля стоков. В данной работе точка зрения иная. Мы предлагаем формулировать его для химпотенциала в виде $\left.\beta \mu\right|_{S_{e x t}}=\ln \left(\bar{C} / C^{e}\right)$. Это стандартный вид химпотенциала ТД в эффективной среде, где влияние конкретного стока нивелируется всем ансамблем. Тогда

$$
\left.C(\mathbf{r}) \exp \left(E_{\text {int }}(\mathbf{r})\right)\right|_{S_{\text {ext }}}=\bar{C} .
$$

В терминах новой переменной

$$
\psi(r, z)=\left[C(r, z) \exp E_{\text {int }}(r, z)-C_{R}\right] /\left[\bar{C}-C_{R}\right]
$$

квазистационарное уравнение диффузии в безразмерных цилиндрических координатах с учетом изотропии в базисной плоскости имеет вид

$$
\frac{\partial^{2} \psi}{\partial r^{2}}+\frac{\partial^{2} \psi}{\partial z^{2}}+\left(\frac{1}{r}-\frac{\partial E_{\text {int }}}{\partial r}\right) \frac{\partial \psi}{\partial r}-\frac{\partial E_{\text {int }}}{\partial z} \frac{\partial \psi}{\partial z}=0
$$

с граничными условиями на внутренней и внешней тороидальных поверхностях

$\psi(r, z)=0$ на $\left(r^{2}+z^{2}+R^{2}-r_{c}^{2}\right)^{2}=4 R^{2} r^{2}$

$$
R-r_{c} \leq r \leq R+r_{c}
$$

$\psi(r, z)=1$ на $\left(r^{2}+z^{2}+R^{2}-R_{\text {ext }}^{2}\right)^{2}=4 R^{2} r^{2}$

$$
\begin{gathered}
R-R_{\text {ext }} \leq r \leq R+R_{\text {ext }} \text { для } R>R_{\text {ext }} ; \\
0 \leq r \leq R+R_{\text {ext }} \text { для } R<R_{\text {ext }} .
\end{gathered}
$$

Для искомого потока тогда имеем:

$$
\begin{gathered}
J=2 \pi R \frac{D}{\omega} Z\left(r_{c}, R, R_{\text {ext }}\right)\left[\bar{C}-C_{R}\right], \\
Z\left(r_{c}, R, R_{\text {ext }}\right)=\frac{1}{2 \pi R} \iint_{S} \exp \left(-E_{\text {int }}(r, z)\right)[\mathbf{n} \nabla \psi(r, z)] d \sigma .
\end{gathered}
$$

Безразмерную величину $Z\left(r_{c}, R, R_{\text {ext }}\right)$ называют эффективностью поглощения ТД дислокационной петлей, а величину $B=1-Z_{v} / Z_{i}$ - ее фактором предпочтения (bias), где индексами $v, i$ обозначены вакансии и СМА соответственно. Если $B>0$, говорят, что петля имеет предпочтение к междоузлиям. Диффузионная задача $(10),(11)$ решалась численно методом конечных разностей $[7,19]$. На рис. 3 показано сечение тороидального резервуара, содержащего петлю, с учетом симметрии отражения в плоскости $x=0$ и симметрии относительно поворота вокруг оси oz. Для радиуса $R>R_{\text {ext }}$ диффузионное поле рассчитывалось в области, ограниченной поверхностями $\mathrm{DA}, \mathrm{AB}, \mathrm{BC}, \mathrm{CD}$, для $R<R_{\text {ext }}$ - поверхностями ОА, АВ, $\mathrm{BC}, \mathrm{CD}$, DO. Указанная симметрия накладывает дополнительные граничные условия: $\partial \psi / \partial z=0$ на $\mathrm{DA}, \mathrm{BC}, \mathrm{OA}$, соответствующее нулевому потоку через плоскость $z=0$, и $\partial \psi / \partial r=0$ на DO (ось симметрии). После этого по формуле (12) вычислялась эффективность поглощения ТД $\alpha$-го сорта и фактор предпочтения $B$. Внутренняя произвольная поверхность $S$ в (12) для удобства вычислений выбрана в виде прямоугольника вращения. На рис. 3 это контур $L$. Расчеты выполнены для циркония, материальные параметры которого приведены выше. Точечный дефект моделируется центром дилатации.

\section{4. Результаты и их обсуждение}

На рис. 4 представлена зависимость bias-фактора дислокационных петель разной природы от их радиуса в единицах $b(+-$ вакансионная петля, о - междоузельная петля; $\left.r_{c}=3 b\right)$. Для упрощения вычислений радиус сечения внешнего тора $R_{\text {ext }}$ задавался одинаковым для вакансий и СМА, что соответствует приближению $\rho \approx 1 / \pi R_{e x t}^{2}$ (где $\rho-$ плотность стоков). Если доминирующим стоком в системе являются дислокации, то значение $R_{\text {ext }}=125 b$ - соответствует плотности дислокаций $\rho \approx 2 \cdot 10^{10} \mathrm{~cm}^{-2}$ рис. $4, a$, а $R_{\text {ext }}=55 b-$ плотности дислокаций рис. $4, b$. Штриховая линия соответствует пределу прямолинейной дислокации $B_{s t r}$. Надо сказать, что аналогичная задача решалась многими авторами, правда в приближении упруго изотропной 

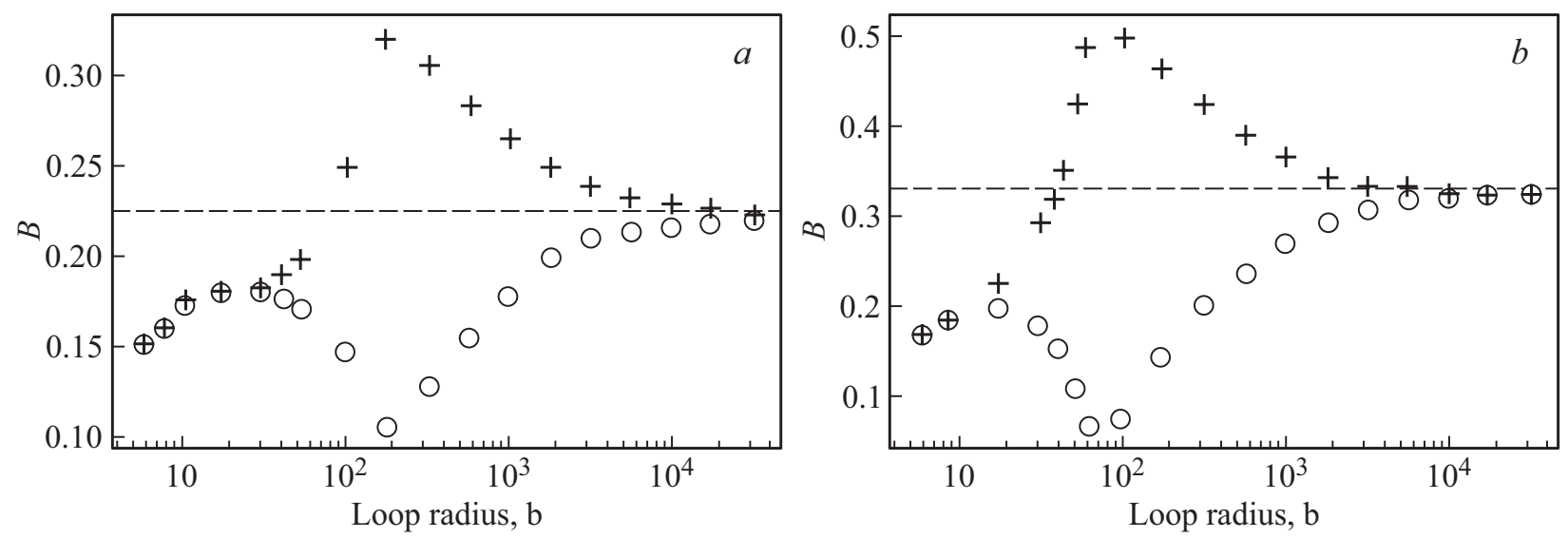

Рис. 4. Зависимость bias-фактора $B$ от радиуса петли для $R_{e x t}=125 b(a)$ и $R_{e x t}=55 b(b)$ (о - междоузельная петля, +вакансионная).

среды, а также в цилиндрическом или сферическом резервуаре. Основные результаты этих исследований сформулированы в [7]. Естественно сравнить их с нашими. Во-первых, отмечается, что дислокационные петли являются „biased“-стоками, которые эффективнее поглощают СМА, чем вакансии. Этот вывод подтверждается и нашими расчетами, поскольку $B>0$ для обоих видов петель. Во-вторых, что фактор предпочтения зависит от радиуса петли и плотности стоков. И это абсолютно верно. Однако далее следует важный вывод о том, что фактор предпочтения не зависит от природы петли, причем для любого вида резервуара. В нашем случае „традиционная“ симметрия в поглощении ТД петлями разной природы нарушается: bias-фактор междоузельной петли при фиксированном внешнем радиусе тора $R_{\text {ext }}$ имеет минимум с выходом на соответствующие значения прямолинейной дислокации. Кстати, последнее обстоятельство характерно именно для тороидального резервуара, в случае сферы или цилиндра $B$ монотонно растет. Аналогичная зависимость для вакансионной петли демонстрируют наличие максимума. Положения минимума и максимума смещаются в область меньших размеров петель с увеличением плотности стоков или с уменьшением $R_{\text {ext }}$. Обратим внимание, что фактор предпочтения петель разной природы с ростом их размера выходит на одно и то же значение, как по идее и должно быть. Формальная причина нарушения указанной симметрии связана с граничным условием на внешнем радиусе тора $R_{\text {ext }}(11)$, поскольку численный анализ уравнений $(10)-(12)$ показал, что учет упругой анизотропии кристалла (5), (6) для базисных краевых петель существенной роли не играет. В нашем случае на границе области влияния стока и эффективной среды предполагается равенство химпотенциалов ТД. В результате мы имеем одну эффективность поглощения $Z\left(r_{c}, R, R_{e x t}\right)$, поток на петлю, пропорциональный разности $\left[\bar{C}-C_{R}\right]$, и граничное условие $(11)(\psi=1)$, не чувствительное к типу петли. В работах других авторов (см. например [7]) традиционно предполагается равенство концентраций ТД $\left(C\left(\left.\bar{r}\right|_{r=R_{e x t}}=\bar{C}\right)\right.$. Тогда возникают две эффективности: поглощения и испускания, соответственно два потока, граничное условие для расчета потока поглощения принимает вид $\psi=\bar{C} \exp \left(E\left(R_{e x t}\right)\right)$, и как результат - независимость bias-фактора поглощения от природы петли. Ответить однозначно на вопрос, какой подход правильный, пока не удается. Обнадеживающим является то обстоятельство, что в нашем варианте, базисные междоузельные петли, обладающие наименьшим фактором предпочтения, можно считать основным стоком для вакансий. Поэтому они не имеют шансов на выживание, что и наблюдается экспериментально. Судьба вакансионных петель неоднозначна. Большие петли не могут выживать из-за их большего bias-фактора по сравнению с прямолинейной дислокацией, однако именно они и наблюдаются в процессе радиационного роста циркония. Их „точкой накопления“ можно считать размер, когда $B=B_{s t r}$. Однако, если в процессе эволюции микроструктуры кристалла средний biasфактор системы стоков в целом станет больше, чем у прямолинейной дислокации и будет расти, то „точка накопления“ может смещаться в сторону увеличения размеров выживающих вакансионных петель. Другими словами необходим „внешний“ источник вакансий в базисную плоскость. Таковым могут быть междоузельные петли, зарождающиеся на призматических плоскостях при радиационном росте циркония. Однако аналогичный анализ их фактора предпочтения это предмет будущих исследований.

\section{Финансирование работы}

Работа выполнена за счет средств бюджетной программы Украины „Поддержка развития приоритетных направлений научных исследований“ (КПВК 6541230).

\section{Конфликт интересов}

Авторы заявляют, что у них нет конфликта интересов. 


\section{Приложение}

$$
\begin{aligned}
& V\left(\tau_{3}^{2}\right) \equiv\left(1-3 \tau_{3}^{2}\right) \Phi\left(\tau_{3}^{2}\right)+2 \tau_{3}^{2}\left(1-\tau_{3}^{2}\right) \frac{d \Phi}{d \tau_{3}^{2}} \\
& W\left(\tau_{3}^{2}\right) \equiv F\left(\tau_{3}^{2}\right)-2\left(1-\tau_{3}^{2}\right) \frac{d F}{d \tau_{3}^{2}} \\
& \Phi\left(\tau_{3}^{2}\right) \equiv \frac{2 i}{\left(z_{1}+z_{2}\right)} \frac{a+b+\chi+\rho}{A\left(\tau_{3}^{2}\right)} ; \\
& F\left(\tau_{3}^{2}\right) \equiv \frac{2 i}{\left(z_{1}+z_{2}\right) A\left(\tau_{3}^{2}\right)} \\
& \times\left((b+\rho)+(a+b-\rho) \tau_{3}^{2}-\frac{a+2 b}{z_{1} z_{2}}\right) ; \\
& \mathrm{K}\left(\tau_{3}^{2}\right) \equiv \mathrm{N}\left(\tau_{3}^{2}\right)-2 \tau_{3}^{2} \frac{d \mathrm{~N}}{d \tau_{3}^{2}}-3 \tau_{3}^{2} \mathrm{M}\left(\tau_{3}^{2}\right) \\
& +2 \tau_{3}^{2}\left(1-\tau_{3}^{2}\right) \frac{d \mathrm{M}}{d \tau_{3}^{2}} \\
& \mathrm{~N}\left(\tau_{3}^{2}\right) \equiv \frac{2 i}{\left(z_{1}+z_{2}\right)} \frac{R\left(\tau_{3}^{2}\right)}{b A\left(\tau_{3}^{2}\right)}-\frac{b \tau_{3}^{2}}{\sqrt{b P\left(\tau_{3}^{2}\right)}(b+\rho)\left(1-\tau_{3}^{2}\right)} ; \\
& \mathrm{M}\left(\tau_{3}^{2}\right) \equiv \frac{2 i}{\left(z_{1}+z_{2}\right)} \frac{S\left(\tau_{3}^{2}\right)}{\left(1-\tau_{3}^{2}\right) b A\left(\tau_{3}^{2}\right)} \\
& -\frac{P\left(\tau_{3}^{2}\right)+b \tau_{3}^{2}}{\sqrt{b P\left(\tau_{3}^{2}\right)}(b+\rho)\left(1-\tau_{3}^{2}\right)^{2}} . \\
& R\left(\tau_{3}^{2}\right)=\frac{(a+b)(b+\rho)}{z_{1} z_{2}}-\frac{A\left(\tau_{3}^{2}\right) P\left(\tau_{3}^{2}\right)}{2(b+\rho)\left(1-\tau_{3}^{2}\right)} \\
& \times\left(z_{1} z_{2}-\frac{b}{P\left(\tau_{3}^{2}\right)}\right) \\
& S\left(\tau_{3}^{2}\right)=(a+b)(b+\rho)\left[\frac{1}{z_{1} z_{2}}+\tau_{3}^{2}\right]-\frac{A\left(\tau_{3}^{2}\right) P\left(\tau_{3}^{2}\right)}{2(b+\rho)\left(1-\tau_{3}^{2}\right)} \\
& \times\left[\left(z_{1} z_{2}-\frac{b}{P}\right)+\left(z_{1}^{2} z_{2}^{2}+\frac{b}{P}\left[z_{1} z_{2}-\frac{2 B}{A}\right]\right) \tau_{3}^{2}\right] . \\
& A\left(\tau_{3}^{2}\right)=2\left[k+l\left(1-\tau_{3}^{2}\right)-m\left(1-\tau_{3}^{2}\right)^{2}\right] ; \\
& B\left(\tau_{3}^{2}\right)=2 k+l\left(1-\tau_{3}^{2}\right) ; \\
& P\left(\tau_{3}^{2}\right)=b+\rho\left(1-\tau_{3}^{2}\right) \text {. } \\
& k=(a+2 b)(b+\rho) \text {; } \\
& l=(a+2 b) \gamma+(2 b-\chi)(\chi+2 \rho) \text {; } \\
& a=C_{12} ; \\
& b=\frac{1}{2}\left(C_{11}-C_{12}\right)=C_{66} ; \\
& \chi=C_{13}-C_{12} ; \gamma=C_{11}+C_{33}-4 C_{44}-2 C_{13} \text {; }
\end{aligned}
$$

$$
\begin{gathered}
\rho=C_{44}-\frac{1}{2}\left(C_{11}-C_{12}\right) \\
z_{1} z_{2}=-\sqrt{\frac{2 k}{A\left(\tau_{3}^{2}\right)}} \\
z_{1}+z_{2}=i \sqrt{2}\left(\sqrt{\frac{2 k}{A\left(\tau_{3}^{2}\right)}}+\frac{B\left(\tau_{3}^{2}\right)}{A\left(\tau_{3}^{2}\right)}\right)^{1 / 2} .
\end{gathered}
$$

\section{Список литературы}

[1] A.D. Brailsford, R. Bullougb. J. Nucl. Mater. 44, 121 (1972).

[2] A.D. Brailsford, R. Bullougb , M.R. Hayns. J. Nucl. Mater. 60, 246 (1976).

[3] V.A. Borodin, A.E. Volkov, A.I. Ryazanov. J. Nucl. Mater. 307-311, 862 (2002).

[4] M. Griffiths. J. Nucl. Mater. 159, 190 (1988).

[5] C.H. Woo. J. Nucl. Mater. 276, 90 (2000).

[6] S.N. Buckley. Properties of Reactor Materials and Effects of Irradiation Damage. Butterworths, London (1962). 413 p.

[7] V.I. Dubinko, A.S. Abyzov, A.A. Turkin. J. Nucl. Mater. 336, 11 (2005).

[8] C.H. Woo, U. Gosele. J. Nucl. Mater. 119, 219 (1983).

[9] C.H. Woo. J. Nucl. Mater. 159, 237 (1988).

[10] G.D. Samolyuk, A.V. Barashev, S.I. Golubov, Y.N. Osetsky, R.E. Stoller. Acta Mater. 78, 173 (2014).

[11] Дж. Эшелби. Континуальная теория дислокаций. Наука, M. (1963). 215 c.

[12] А.М. Косевич. Физическая механика реальных кристаллов. Наук. думка, Киев. (1981). 328 с.

[13] E. Kröner. Z. Phyz. 136, 402 (1953).

[14] M.H. Yoo. Phys. Status Solidi B 61, 411 (1974).

[15] П.Н. Остапчук, О.Г. Троценко. ФТТ 58, 1749 (2016).

[16] П.Н. Остапчук, О.Г. Троценко. ФТТ 59, 912 (2017).

[17] L. Fast, J.M. Wills, B. Johansson, O. Eriksson. Phys. Rev. B 51, 17431 (1995).

[18] А.М. Косевич, З.К. Саралидзе, В.В. Слезов. ФТТ 6, 3383 (1964).

[19] C.H. Woo, W.S. Liu, M.S. Wuschke. AECL-6441 (1979).

Редактор Т.Н. Василевская 